\title{
Allen Frances y la pureza del diagnóstico.
}

Allen Frances and the purity of diagnosis.

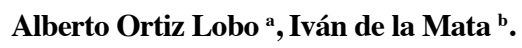

${ }^{a}$ Psiquiatra, Centro de Salud Mental "Salamanca". Hospital Universitario La Princesa, Madrid, España. ${ }^{b}$ Psiquiatra, Centro de Salud Mental de Leganés. Instituto Psiquiátrico José Germain, Leganés, Madrid, España.

Correspondencia: Alberto Ortiz Lobo (aortizlobo@yahoo.es)

Allen Frances es un psiquiatra americano, nacido en 1942 y en la actualidad el jefe de departamento de psiquiatría de la Universidad de Duke, en Carolina del Norte. Es mundialmente conocido por presidir el grupo de trabajo que escribió el DSM-IV y, posteriormente, por ser la voz referente en la crítica al DSM-5. Fruto de esta crítica, es su libro que se ha traducido recientemente al castellano por la editorial Ariel: ¿Somos todos enfermos mentales? Manifiesto contra los abusos de la psiquiatría. Precisamente, con motivo de la presentación de su obra en nuestro país, apareció en diversos medios de comunicación y pudimos entrevistarlo para la AEN.

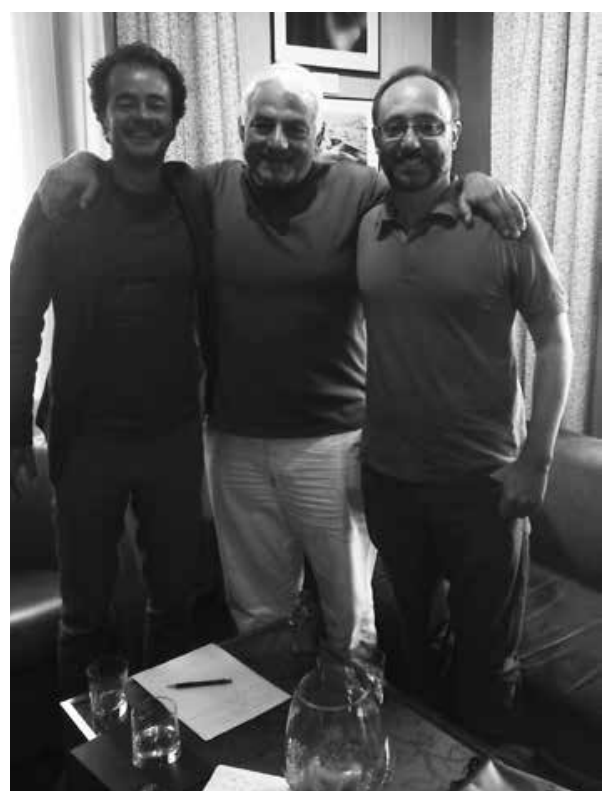

¿Cuáles considera que son los mayores peligros del DSM-5?

Ya estamos atravesando un periodo de inflación de diagnósticos en psiquiatría, con tratamientos excesivos, se le da medicación a la gente que realmente no lo necesita. El DSM-5 ha introducido una cantidad de diagnósticos nuevos que amenazan convertir la inflación diagnóstica en hiperinflación diagnóstica. El duelo normal se convierte en un trastorno depresivo mayor, el hecho de que se me olviden las cosas porque ya tengo una edad, se ve como predemencia, como un trastorno cognitivo menor. El que yo desayune a lo grande, se vuelve un trastorno por atracón. Las rabietas de mis nietos se convierten en un trastorno de disregulación emocional. Si una persona tiene preocupaciones por un síntoma físico, tiene un trastorno por síntomas somáticos. El TDAH, que ya antes se sobrediagnosticaba, será cada vez más común con el DSM-5. A la vez que estamos sobretratando a las personas que no lo nece- 
sitan, estamos ignorando terriblemente a los que están gravemente enfermos, que desesperadamente necesitan mayor acceso al cuidado y una vivienda digna. Cuanto más expandimos el sistema para incluir a las personas sanas "preocupadas", menos recursos tenemos para cuidar a los que de verdad están enfermos.

Usted ha participado en el DSM-III, en el DSM-III-R, en el DSM-IV, ¿cómo se construye un nuevo diagnóstico? ¿Cómo es el proceso? ¿Qué agentes intervienen? ¿Cómo se fundamenta?

Desgraciadamente en la psiquiatría no tenemos pruebas biológicas, los diagnósticos se basan en juicios falibles y subjetivos. El hecho de que haya un conjunto de criterios definitivos por lo menos nos da la oportunidad de conseguir fiabilidad entre los distintos clínicos que ven al mismo paciente. Pero la pregunta es: ¿qué diagnósticos deberían ser incluidos dentro del sistema?, ¿cuáles deberían ser los márgenes para definirlos? La única manera de hacerlo, en este punto, es tener grupos de expertos que revisen la literatura disponible y lleguen a algún tipo de consenso sobre cómo definir mejor los diferentes trastornos mentales y cuáles merecen estar en el manual. El problema es que no hay una línea clara que separe los trastornos mentales de la normalidad, sobre todo los trastornos más leves. Todo el mundo puede estar de acuerdo en cuáles son los trastornos graves y tampoco hay tanta polémica respecto a cómo definirlos, pero cuando se trata de los trastornos más leves, hay un límite muy borroso con la normalidad y pequeños cambios en la definición puede incluir como pacientes a millones de personas que previamente eran consideradas como normales, de manera que el límite entre lo normal y la enfermedad mental es a la vez borroso y muy "poblado". Los expertos en cualquier área casi siempre quieren expandir las fronteras de los trastornos de su especialidad. He estado trabajando con expertos desde 1978, cuando empezamos a preparar el DSM-III, miles de expertos, ni uno jamás vino y me dijo: "vamos a reducir mi diagnóstico". Todos sobrevaloran su área, toman decisiones basadas en lo que funciona para ellos en clínicas especializadas, en hospitales de la universidad y no entienden que la mayoría de los fármacos psiquiátricos son recetados por los médicos de atención primaria normalmente, después de citas que duran siete minutos, de pacientes que apenas conocen y que además están viniendo a la consulta en el peor día de sus vidas. La mayoría mejorarían solos, con placebo, con tiempo, con resiliencia, apoyo, reducción del estrés... Los médicos de atención primaria están muy influenciados por el comercial de la farmacéutica y la forma más fácil de sacar al paciente de la consulta es darle una pastilla, incluso cuando esa pastilla a largo plazo puede causarle más daño que beneficio. De manera que es muy importante convencer a los expertos para reducir su exuberancia diagnóstica, para hacer que el sistema sea conservador. Intentamos hacer esto con el DSM-IV y rechazamos todas menos dos de las 92 sugerencias de nuevos diagnósticos. Cuando comple- 
PARA EL DEBATE

tamos el manual estábamos muy contentos, habíamos mantenido nuestra posición en contra de la inflación diagnóstica, pero nos equivocamos: en los últimos 20 años ha habido un aumento dramático en la prevalencia del TDAH. de autismo, de trastorno afectivo bipolar... Esto no es porque la gente haya cambiado, o porque el manual haya cambiado, aunque fue parte del problema, el tema principal fue cómo se utilizó ese manual y hay fuerzas externas muy poderosas que empujan hacia el mal uso del manual, que empujan hacia el diagnóstico excesivo, y si hay cualquier cosa dentro del manual que pueda ser mal utilizado, descubrimos que se va a utilizar erróneamente. Lo que yo creo que se necesita para el futuro es una aplicación más cautelosa del diagnóstico, especialmente para los problemas más leves de la vida. España es un buen ejemplo: en cualquier país que tenga una tasa de paro del $25 \%$, que no haya trabajo para la gente que sale de la universidad, que haya una gran incertidumbre sobre el futuro, muchísima gente va a experimentar síntomas de tristeza y ansiedad, eso es la naturaleza humana. La condición humana es preocuparse y sentirse triste en tiempos tristes, eso es normal, eso no es un trastorno mental. Pero la tendencia es redefinirlo como trastorno mental en el individuo más que verlo como un problema de la sociedad, medicar al individuo y darle fármacos. Pero de hecho, la tasa de respuesta al placebo de este tipo de reacción al estrés social es de más del $50 \%$, casi tanta como la tasa de respuesta a la medicación. La medicación no suele ayudar, pero cuando el paciente se siente mejor con el paso del tiempo, después de haber visto al médico en el peor día de su vida, va a atribuir la mejoría a la medicación y a menudo se quedará con ella durante periodos muy largos de tiempo cuando tal vez no está ayudando y puede tener efectos secundarios y complicaciones. Cuando nos estamos gastando todo este dinero en fármacos no nos estamos gastando el dinero en apoyo social ni en tratar a los que están gravemente enfermos. Las farmacéuticas se benefician de esta definición que amplía el trastorno mental y su márketing está diseñado para vender la enfermedad, para aumentar el uso inapropiado de la medicación, pero es malo para los individuos que se la toman cuando no se necesita, es malo para la sociedad. No quiero que nadie que escuche esto piense que estoy en contra de la medicación, yo, de hecho, estoy muy a favor de la medicación para un 5-10\% de la población que realmente la necesita, pero en EE. UU hay un $20 \%$ de la población que está recibiendo medicación y me han contado que en España hay incluso un porcentaje más alto que está recibiendo medicinas psiquiátricas. Deberíamos centrarnos en la gente que lo necesita y que a menudo no tiene acceso al tratamiento, que no tiene vivienda digna. En España tenéis un soporte social mejor, un sentido de la familia que da soporte que no lo tenemos en EE. UU. En EE. UU. la desinstitucionalización ha sido una catástrofe absoluta para los pacientes psiquiátricos. Hemos cerrado un millón de camas en los últimos 40 años en los hospitales psiquiátricos y ahora tenemos un millón de pacientes psiquiátricos que están en las cárceles y cientos de miles de pacientes psi- 
quiátricos "sin techo" también. Así que hay una paradoja muy cruel de sobretratar a la gente que no lo necesita y vergonzosamente ignorar a la gente que sí lo necesita. Vosotros estáis en mejor posición, no nos copiéis.

Volviendo al tema de los expertos en los manuales diagnósticos, ¿nos podría hablar de los conflictos de intereses que hay en los paneles de expertos en la elaboración de los manuales?

Ha habido mucho debate sobre el hecho de que, como la mitad de la gente que trabaja en el DSM ha tenido alguna conexión con las farmacéuticas, hay una preocupación de que ese conflicto de intereses financiero afectaría a las decisiones que se tomaban. Yo conozco a estas personas y ellos no tienen ningún deseo de ayudar a las farmacéuticas. Es verdad que han tomado decisiones muy terribles, pero con "corazones puros". Pero hay otros dos tipos de conflictos que son muy importantes: el primero es el conflicto de intereses intelectuales de los expertos y las ataduras emocionales a sus áreas que he mencionado antes. El segundo es el conflicto de interés de la APA, que es financiero y por supuesto depende mucho de los beneficios. El DSM es un "best seller" y se venden cientos de miles de copias en EE. UU. cada año. La APA se apresuró en llevar a la prensa el DSM-5, de una manera muy brusca, antes de que realmente estuviese cuidadosamente preparado, en parte porque su presupuesto dependía de que eso apareciera en cierto momento para cubrir déficits que de otra manera hubieran resultado problemáticos. También teníamos ambiciones muy altas para el DSM-5, para que fuera un cambio de paradigma dentro de la psiquiatría. Estas ambiciones llevaron a muchos de los problemas en su preparación y parte de la ambición puede que haya sido para mejorar las ventas haciendo que el DSM-5 pareciese más especial que cualquier clasificación psiquiátrica de lo que podía haber sido en ese momento. Ha habido muy poco avance científico para apoyar cambios dramáticos en el sistema diagnóstico. En los últimos 40 años hemos presenciado descubrimientos apasionantes sobre cómo funciona el cerebro humano y la genética que subyace en la naturaleza humana. Pero esta emoción que se ha sentido en la neurociencia y en la genética no se ha traducido en una mejora de la práctica clínica: no hay un solo paciente al que se le haya ayudado, únicamente está lo que hemos aprendido en los estudios de ciencia básica. La traslación desde la ciencia básica a la práctica clínica es difícil en toda la medicina, pero esto es especialmente complicado en la psiquiatría porque el cerebro es la cosa más compleja del universo conocido, estas tres libras que llevamos aquí dentro contiene más neuronas que estrellas en la galaxia y cada una de ellas tiene mil conexiones, cientos de neurotransmisores que median su comunicación. El cerebro no revela sus secretos rápidamente, cuanto más aprendemos, menos sabemos. Recientemente se ha dicho que hay más de cien genes para la esquizofrenia, esto quiere decir que es muy complicado comprender la genética de la esquizofrenia. No hay una forma única de esquizofrenia. Tolstoi dijo que 
PARA EL DEBATE

todas las familias son felices de la misma manera pero cada familia infeliz lo es a su propia manera. Es mucho más fácil para nosotros aprender sobre el funcionamiento normal del cerebro que entender las maneras en las que el cerebro falla. Considerando la complejidad del cerebro es increíble que funcionemos lo bien que lo hacemos. No habrá una esquizofrenia, habrá cientos de esquizofrenias y no hay un cáncer de mama, hay cientos de cánceres diferentes, y el pecho es el órgano más sencillo del cuerpo, el cerebro es mucho más complejo. No nos debería sorprender si tardamos décadas en dar pasos muy pequeñitos para entender la patogénesis de los trastornos psiquiátricos, pero tenemos que cuidar a nuestros pacientes ahora, no podemos esperar a que las neurociencias nos proporcionen pequeñas respuestas durante largas décadas. El enfoque debería estar en lo que sí que sabemos y sabemos mucho, en aplicar las herramientas que sí que tenemos y la verdad es que tenemos herramientas muy potentes y no requiere una ciencia muy compleja el diseñar un buen tratamiento, solo requiere financiación para hacer las cosas correctas, controlar las compañías farmacéuticas que quieren hacer las cosas incorrectas y reeducar a los psiquiatras, pero sobre todo, lo más importante, a los médicos de atención primaria, en los problemas que tienen que ser tratados y los problemas que tienen que ser dejados en paz.

Nos preguntábamos, al hilo de lo que estaba comentando de la diversidad de la expresión de la enfermedad mental, ¿cuáles serían los pros y los contras de los diagnósticos categoriales?

Con el tiempo, el diagnóstico dimensional será muy superior y más preciso a la hora de describir fenómenos que responden a un continuum largo, sin una línea clara de demarcación. Así, utilizamos una medición dimensional para el peso y la altura y los trastornos psiquiátricos tampoco tienen fronteras claras, así que tiene sentido tener un enfoque dimensional a la hora de enfocarlos. Pero las dimensiones son mucho más complejas y los humanos están "cableados" para pensar más en nombrar las cosas que en numerarlas. A los ordenadores les encantan los números, a los clínicos humanos les gustan los nombres: "esto es azul". Pero esta es una descripción muy imprecisa porque hay tantos tonos de azul... Si estuviéramos haciendo un experimento físico, sería mejor dar el número de la longitud de onda, pero en cada conversación es más fácil dar el nombre. El DSM-5 intentó ser más dimensional, pero el problema es que no había ningún sistema organizado en dimensiones y un sistema dimensional añadiría una complejidad mayor para los clínicos que ya de por sí no tienen suficiente tiempo para conocer a sus pacientes. Los esfuerzos de los expertos del DSM-5 para crear dimensiones fallaron y estas no se incluyeron en el sistema. Mi esperanza es que gradualmente el diagnóstico dimensional se convierta en una parte integral de la práctica diaria, pero va a ser un proceso lento. En teoría es una idea maravillosa, pero en la práctica es difícil de incorporar. 
El DSM ha sido criticado desde dos posiciones, por un lado el National Institute of Mental Health (NIMH) que rechaza el DSM como manual para sus investigaciones y por otro lado desde posiciones más psicológicas (como la División de Psicología del Colegio de Psicólogos del Reino Unido) o desde movimientos de usuarios como Hearing Voices, ¿cuál es su posición respecto de estas dos críticas contrapuestas?

Yo he sido tan crítico del DSM como cualquiera, pero también comprendo los motivos por los que, con todas sus limitaciones, tiene una utilidad. La crítica del NIMH es válida en el sentido de que los últimos 30 años de estudios de los diagnósticos actuales han sido muy decepcionantes. Había mucho optimismo en que íbamos a obtener respuestas biológicas simples y no las tenemos. El NIMH está intentado renovar desesperadamente el esfuerzo estudiando correlaciones biológicas de cosas más simples que los complejos síndromes del DSM. Quizás es muy difícil entender la esquizofrenia, pero quizás se puedan entender las alucinaciones. Esa ya es una estrategia muy válida, aunque ha sido sobreestimada, pero eso no quiere decir que podamos usar el método del NIMH, que básicamente es un método de investigación, para tratar a los pacientes. La crítica del NIMH es una crítica válida desde el campo de la investigación, pero no es una crítica válida al DSM como herramienta clínica porque ahora mismo es la única herramienta clínica que tenemos.

Los psicólogos han criticado igualmente el DSM desde la otra posición, señalando que no presta suficiente atención al contexto psicosocial y esto es también una crítica válida, pero la psicología no ofrecen otro método que pueda reemplazarlo. Es muy fácil criticar el DSM, a mí también se me da bien, pero es muy difícil mejorarlo.

Desde movimientos de usuarios se reivindica su papel de expertos a la hora de conceptualizar los diagnósticos o las enfermedades como por ejemplo Hearing voices ¿que nos puede decir de esto?

Hearing Voices es un interesante ejemplo. Hay una persona maravillosa en este movimiento con la que he escrito varios blogs juntos, Eleanor Longden. El punto más importante que yo aclararía es que no tiene sentido ser un seguidor ciego de lo que hace la psiquiatría, porque muchas veces algo de lo que hacemos no lo hacemos bien y tenemos que reconocer que a muchos pacientes les ha ido mal, incluso, y han sido dañados por la psiquiatría. He visto otros muchos a los que les hemos ayudado pero cientos están peor después de ser tratados. Pero, por otro lado, no creo que podamos aceptar los puntos de vista de aquellos que se oponen por completo a la psiquiatría. Muchas de estas personas son aquellas que menos se beneficiaron de la psiquiatría o se sintieron dañadas. Es razonable su malestar, pero lo que no se dan cuenta es de que su experiencia personal no puede ser la experiencia universal, no puede generalizarse, que ellos pueden haber sido decepcionados, 
PARA EL DEBATE

pero no todos. Es decir, no es una cuestión de estar totalmente a favor o totalmente en contra de la psiquiatría. La psiquiatría bien aplicada puede ser maravillosa y la psiquiatría aplicada mal puede causar daño. La gente razonable que critica la psiquiatría como Eleanor Longden comprende esto y es posible encontrar en nuestros blogs conjuntos una base común para entendernos. Ella está muy interesada en la no-medicación, en el cuidado de los pacientes porque eso fue muy importante para ella misma después de una enfermedad psiquiátrica muy grave: aprender a vivir con las voces en lugar de suprimirlas. Yo también creo que las intervenciones psicosociales son una parte muy importante del tratamiento de los trastornos mentales graves pero creo que la medicación es importante y ella reconoce que para algunas personas también lo es. Yo apoyo mucho la libertad individual, que los pacientes tomen sus decisiones, que negocien un plan de tratamiento, que en parte lo estén haciendo ellos... A mí no me gusta el tratamiento coercitivo, pero hay algunas situaciones en que es absolutamente necesario: algunas personas sino reciben tratamiento tendrían unas vidas muchísimo peores y ella eso también lo entiende, que cuando estamos tratando de casos individuales puede haber muchas más acuerdo que si hablamos de forma abstracta. La gente razonable tiene muchas más posibilidades de estar de acuerdo en cómo tratar con problemas prácticos, incluso si parten de posiciones ideológicas diferentes. Es importante que la ideología no nos ciegue el sentido común.

Desarrollando este aspecto, porque le he entendido que, de alguna manera, la medicalización es debido en parte al poder de la psiquiatría y de las asociaciones profesionales, me preguntaba si una mayor participación de los usuarios en la investigación, diseño de servicios e incluso en la elaboración de los diagnósticos influiría en la disminución del daño que se hace a estas personas.

Creo que el modelo de la psiquiatría solía ser bio-psico-social, intentando mirar al paciente de una manera global y entender los problemas en el contexto más amplio posible. El modelo de la psiquiatría ahora se ha vuelto mucho más bio-bio-bio, con menos tiempo e interés por los factores psicológicos o sociales que están involucrados en la causa del problema. La investigación en salud mental, sobre todo la que hace el NIMH, se ha vuelto completamente biológica. Es una investigación sobre el cerebro, no de salud mental, todo el dinero va a la neurociencia y esperan que en el futuro haya respuestas biológicas más contundentes, pero no sirven para nada a la hora de cuidar a los pacientes en el presente. Debemos tener investigación biológica, pero también deberíamos saber por qué nuestros pacientes están en la cárcel y cómo sacarlos de ahí. Los grupos de consumidores están muy decepcionados. Ellos ahora suelen centrarse en los que están menos graves. En sus inicios comenzaron con los enfermos más graves, pero éstos son un grupo pequeño, un 5\% quizás. A medida que los grupos de consumidores quieren aumentar su 
población, empiezan a ampliar su margen de interés y acaban perdiendo el foco en los pacientes más graves, los que realmente lo necesitan. También reciben una gran parte de su presupuesto de la industria farmacéutica y a veces parecen que están haciendo más lobby en favor de las farmacéuticas que defendiendo a los pacientes. Así que no es tan simple, la idea de la participación de los consumidores podría ser importante, pero la forma de hacerlo en la práctica puede ser complicada.

Nuestra preocupación es que el DSM-5 sea la última vuelta de tuerca, pero ya el DSM-IV tenía unas prevalencias de enfermedad muy elevadas y creo recordar que el trastorno adaptativo pasó a un segundo lugar en la jerarquía diagnóstica a favor del episodio depresivo mayor, lo que significa que los DSM tiene una perspectiva biológica que poco a poco han dejado de lado lo psicosocial.

Lo que sucedió en este tema, en realidad empezó con el DSM-III, cuando se creó una sola categoría en la que se incluía desde los cuadros más graves hasta los más leves. La expectativa era que el uso de subtipos como la melancolía y utilizando los distintos grados de gravedad se podría encontrar cierta especificidad en lo que era un grupo muy heterogéneo. Pero pasó algo malo, que las compañías farmacéuticas, especialmente después del Prozac, hicieron un marketing muy agresivo de tal manera que dieron el mensaje de que todas las depresiones eran una sola y se debían a un desequilibrio químico, que la depresión estaba infradiagnosticada, y que era muy fácil de diagnosticar. De tal manera que aunque tuvieras una depresión leve o una grave la respuesta siempre era una pastilla. Antes solíamos tener la idea de que había una depresión endógena y unas reactivas y que éstas se podían contextualizar mucho más socialmente y que respondían peor a la medicación y se podían beneficiar de psicoterapia. La decisión del DSM III de juntarlas perdió esta distinción.

\section{éxito? \\ ¿Cuál es el futuro del diagnóstico en psiquiatría, va a morir la psiquiatría de}

Yo creo que siempre será necesaria la psiquiatría, porque no existe otra profesión que tenga la capacidad, el interés y la paciencia de lidiar con la enfermedad mental grave. Tenemos que reconocer que la mayoría de la práctica psiquiátrica se está llevando a cabo por médicos de atención primaria y no por psiquiatras. Se está haciendo de una manera muy descuidada y bajo presiones comerciales de las compañías farmacéuticas. Creo que el futuro de la psiquiatría es apasionante si nos centramos en lo que hacemos mejor: cuidar a los pacientes que más nos necesitan, defender no solo su tratamiento médico sino también sus necesidades de vivienda, su apoyo comunitario. Tenemos tratamientos muy eficaces que pueden ayudarles mucho. También son apasionantes los avances que se han conseguido en psicoterapia. Es importante que los psiquiatras se formen en psicoterapia y forme parte 
PARA EL DEBATE

de nuestras herramientas. No deberíamos quedarnos en hacer una lista de síntomas y de efectos secundarios y darles una pastilla porque eso lo puede hacer un ordenador. Tenemos que cuidar la relación terapéutica. Hipócrates dijo que era más importante conocer a la persona que tiene la enfermedad que la enfermedad que tenía la persona. Si nos mantenemos fuertes con un modelo biopsicosocial y no nos olvidamos de la relación con el paciente, aún con la presión por el diagnostico, la psiquiatría sigue siendo una profesión apasionante, que cuando es agradecida para el médico es útil para el paciente.

\section{Epílogo}

Cuando uno escucha o lee a Allen Frances, es difícil resistirse a suscribir la mayor parte de sus afirmaciones. Es un hombre amable y con un discurso que transmite sensatez y preocupación por los más desfavorecidos. Podría parecer sospechosa su particular trayectoria ideológica, desde su primera participación en el DSM-III, a jefe del DSM-IV e, inopinadamente, a detractor del DSM-5, pero, más allá de esta sospecha, el discurso crítico de Allen Frances puede resultar en ocasiones un tanto cándido y aparente. Llama la atención, por ejemplo, su falta de conciencia de la "colaboración" de los lobbies de la industria farmacéutica con los psiquiatras que elaboran el DSM y los conflictos de intereses que hay al respecto. Sin embargo, la falta de independencia en los paneles de expertos se ha señalado como algo crucial en la expansión de las enfermedades mentales (algo que sucede en el resto de la medicina también) y el consiguiente incremento en la prescripción de psicofármacos ${ }^{1}$. Esta ingenuidad desaparece, en cambio, cuando habla de las asociaciones de usuarios y también de los médicos de atención primaria a quienes sí que considera sensibles a las presiones de la industria farmacéutica y protagonistas de la psiquiatrización. Igualmente, Frances critica la desaparición del enfoque psicosocial en la conceptualización de las enfermedades mentales, pero fue precisamente la publicación en 1980 del DSM-III, el definitivo espaldarazo del modelo biomédico frente a esas perspectivas más psicosociales.

Lo que denuncia Allen Frances en su crítica al DSM-5 es un problema que han tenido todos los manuales en los que él ha participado o liderado, y cuya última edición es simplemente un paso más en la evolución natural de las anteriores en lo que a inflación diagnóstica y modelo biomédico se refiere. En este momento, Fran-

\footnotetext{
${ }^{1}$ Cosgrove, L. Krimsky, S. Wheeler, E.E. Kaitz, J. Greenspan, S.B. DiPentima, N.L. Tripartite Conflicts of Interest and High Stakes Patent Extensions in the DSM-5. Psychother Psychosom 2014;83:106-113. Accesible en: http:// www.karger.com/Article/Pdf/357499
} 
ces apela a la sensatez y a la moderación en la escritura e interpretación del DSM, pero no hay un cuestionamiento profundo del manual, de cómo se elabora, por quién o para qué, de su reduccionismo clínico, su potencial capacidad de estigmatización o sus posibles repercusiones negativas en sociedades no occidentales. Si deseamos profundizar en este análisis, tenemos que leer a otros autores que llevan décadas investigando y trabajando en ello, como por ejemplo Sami Timimi, Joanna Moncrieff, Derek Summerfield, Pat Bracken, Bradley Lewis o Philip Thomas, entre otros.

Sin embargo, Allen Frances ha llegado a donde nadie había sido capaz hasta ahora: está logrando, a través de sus blogs, de entrevistas, de su libro y de aparecer en los medios de comunicación, que los profesionales de la salud mental y buena parte de la ciudadanía conozcan y tomen mayor conciencia del grave problema de la expansión desaforada de la psiquiatría y de la transformación de problemas de la vida cotidiana o de origen socioeconómico en enfermedades mentales. Valga por ello nuestro reconocimiento. 